\title{
Colliding Stellar Winds in O-type Close Binary Systems
}

\author{
D. R. GIES ${ }^{1}$ \& M. S. WIGGS ${ }^{2}$ \\ 1 CHARA, Georgia State University, Atlanta, GA 30303, USA \\ 2 Space Telescope Science Institute, Baltimore, MD, USA
}

\section{INTRODUCTION}

In close binary systems of O-type stars, the individual stellar winds will collide between the stars to form shock fronts (Stevens et al. 1992). Binaries with equally luminous stars will have winds of comparable strength, and the shock will occur near the mid-plane between the stars, but in binaries of unequal luminosity, the interaction will occur along a bow shock wrapped around the star with the weaker wind. The presence of the shock region can be detected through excess X-ray emission (Chlebowski \& Garmany 1990), and orbital phase-related variations in the UV P Cygni lines (Shore \& Brown 1988) and optical emission lines (formed in high density regions of circumstellar gas).

We have begun a search for colliding winds through a study of the optical emission lines and UV P Cygni lines in four massive binaries, AO Cas (Gies \& Wiggs 1991), Plaskett's star = HD 47129 (Wiggs \& Gies 1992), 29 UW CMa, and $\iota$ Ori. The optical observations consist of high $S / N$ spectra of the $\mathrm{H} \alpha$ and He I $\lambda 6678$ region obtained with the University of Texas McDonald Observatory 2.1-m telescope and coudé Reticon system. The UV observations were culled from archival IUE high dispersion spectra of several $\mathrm{P}$ Cygni features (N V $\lambda 1240$, Si IV $\lambda 1400$, C IV $\lambda 1550$ ).

Here we present results for the $\mathrm{H} \alpha$ profiles in the two systems that display the most emission, Plaskett's star and $29 \mathrm{CMa}$. The orbital variations of $\mathrm{H} \alpha$ provide valuable clues on the wind interaction. Furthermore, we find evidence of weak emission with similar orbital variations in other lines (e.g., He I 76678 ) which appear as "pure" absorption features in spectra with lower $S / N$. We caution spectroscopic observers of early-type systems that many lines (particularly low excitation) may be contaminated by circumstellar emission; orbital velocity curves should be constructed using only emission-free lines.

\section{PLASKETT'S STAR (O7.5 I + O6 I)}

The $\mathrm{H} \alpha$ emission profiles are illustrated in Figure 1 (top). The spectra are placed in the upper portion of the diagram so that their continua are aligned with the orbital phase of observation (phase $0.0=$ superior conjunction of the primary star). The lower portion shows the same spectra in a gray-scale representation formed by transforming spectral intensity into gray-scale intensity and interpolating in orbital phase between the observations. The $\mathrm{H} \alpha$ emission is marked by a sharp spike-like feature that approximately follows the motion of the primary star (seen in the He II $\lambda 6527$ line at left). The radial velocity curve for this spike lags the photospheric velocity curve of the primary by 0.066 in phase. We have developed a model for the location of the gas producing the "spike" (Wiggs 

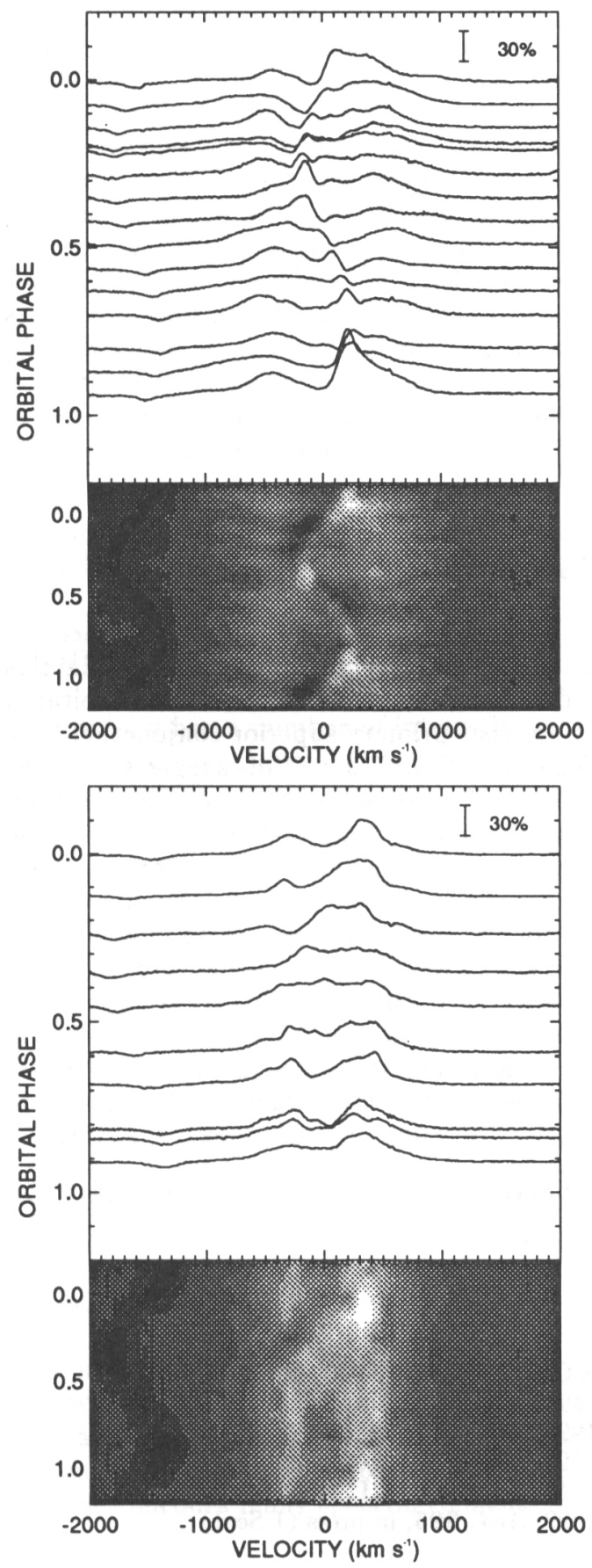

FIGURE 1. H $\alpha$ profiles for (top) Plaskett's Star and (bottom) UW CMa. 
\& Gies 1992) based on the assumption that the observed motion is the vector sum of orbital velocity plus a flow component directed from the secondary (wind dominant) star towards the primary. This model places the emitting gas in an elongated circumstellar cloud centered on the primary. Based on the intensity maxima observed near orbital phases 0.9 and 0.4 , we suggest that the axis of elongation is displaced about $50^{\circ}$ from the axis joining the centers of the two stars. This angle probably corresponds to the Coriolis deflection of a bow shock formed where the wind of the secondary passes the primary, since we find that the UV P Cygni absorption troughs weaken between phases 0.5 and 0.7 when the bow shock occults the secondary's wind. The extreme $\mathrm{H} \alpha$ emission wings undergo nightly intensity variations that display no well-defined orbital motion. We suggest that the high velocity emission is related to instabilities in the intershock region between the two component stars. The $\mathrm{H} \alpha$ spike feature probably forms in post-shock gas while the broad emission component originates close to the shock itself.

\section{29 UW CMA (O8 I + BO I)}

The $\mathrm{H} \alpha$ emission in this system has a very different appearance. The profile (Figure 1, bottom, corrected for the varying continuum level in this partially eclipsing system) is a broad emission feature which shows no orbital motion and attains an intensity maximum near primary superior conjunction (phase 0.13 in the eccentric solution). The lack of orbital motion suggests that the emitting cloud is centered on the center of mass of the system. The stars are in much closer proximity in this system, and we suggest that the wind of the primary star is somewhat focused in the direction of the companion star (see Friend \& Castor 1982) which would lead to increased gas density between the stars. A focused wind may account for the fact that we observe a $P$ Cygni absorption component in $\mathrm{H} \alpha$ during those phases when the hemisphere of the primary facing the companion is partially directed toward us. However a focused wind cannot account for the strong redward emission seen at primary superior conjunction (phase 0.13), and thus the profiles must also have a component from the wind of the secondary star. We suspect that the broad profile represents both gas flowing into and out from the interaction region between the stars.

This research was supported by the NASA Astrophysics Data Program grant NAG 5-1218.

\section{REFERENCES}

Chlebowski, T. \& Garmany, C.D. 1991, ApJ, 368, 241

Friend, D.B. \& Castor, J.I. 1982, $A p J, 261,293$

Gies, D.R. \& Wiggs, M.S. 1991, $A p J, 375,321$

Shore, S.N. \& Brown, D.N. 1988, ApJ, 334, 1021

Stevens, I.R., Blondin, J.M., \& Pollock, A.M.T. 1992, ApJ, 386, 265

Wiggs, M.S. \& Gies, D.R. 1992, $A p J, 396$, in press (1 Sept) 